\title{
RBPBind: Quantitative prediction of Protein-RNA Interactions
}

\author{
Jeff Gaither ${ }^{1, *}$, Yi-Hsuan Lin ${ }^{2}$ and Ralf Bundschuh ${ }^{3, *}$ \\ ${ }^{1}$ Mathematical Biosciences Institute, The Ohio State University, Columbus, 43210, USA, \\ ${ }^{2}$ Department of Biochemistry, University of Toronto, Toronto, Ontario M5S 1A8, Canada and \\ ${ }^{3}$ Department of Physics, Department of Chemistry\&Biochemistry, Division of Hematology, Department of Internal Medicine, Center for \\ RNA Biology, The Ohio State University, Columbus, 43210, USA.
}

*To whom correspondence should be addressed.

\begin{abstract}
Summary: We introduce RBPBind, a web-based tool for the quantitative prediction of RNA-protein interactions. Given a user-specified RNA and a protein selected from a set of several common RNAbinding proteins, RBPBind computes the binding curve and effective binding constant of the reaction in question. The server also computes the probability that, at a given concentration of protein, a protein molecule will bind to any particular nucleotide along the RNA. The software for RBPBind is adapted from the Vienna RNA package, whose source code has been modified to accommodate the effects of single stranded RNA binding proteins. RBPBind thus fully incorporates the effect of RNA secondary structure on protein-RNA interactions.
\end{abstract}

Availability: Our web server is available at http://bioserv.mps.ohio-state.edu/RBPBind

Contact: bundschuh@mps.ohio-state.edu

\section{Introduction}

RNA-binding proteins (or RBPs for short) are the most important functionaries in the process of post-transcriptional regulation. RBPs and other proteins directed by them to particular RNA targets can exert control over the process of translation by recruiting ribosomes to an RNA or preventing their binding to the RNA, by conferring stability or targeting RNAs to rapid decay, and by directing the RNA to specific cellular compartments (Glisovic et al. 2008,

A fundamental question is whether an RBP will bind to an RNA at a given location. In addressing this, both the sequence of bases and the structure of the RNA can be important - and recent technological advances have made it possible to address both. Most notably, RNAcompete (Ray et al. 2009) and RNA Bind-N-Seq (Lambert et al. 2014) use highthroughput approaches to determine the affinity of an RBP for every sequence of a fixed size - e.g. AAAAAA to UUUUUU - and also to establish structural preferences.

However, in vivo a protein interacts with an RNA molecule which might have any number of secondary structures; and structures favorable to protein-binding may not be favorable for base-pairing of the RNA.

To address this complication, we introduce a server, RBPBind, which incorporates both quantitative sequence-dependent protein-binding affinity and the diversity of possible secondary structures which the RNA can present. This enables us to compute the binding curve for an interaction which (as in vivo) typically includes many different secondary structures. We believe RBPBind is unique in its ability to compute the probability of binding for a given RBP with an arbitrary RNA on the basis of both

(c) The Author 2016 experimentally-determined sequence preferences and secondary structure prediction.

RBPBind operates using a modified version of the Vienna RNA package (Lorenz et al. 2011), one of the more widely-used tools for computing RNA secondary structures. Our basic device is to computationally treat RNA-protein interactions as a phenomenon of secondary structure itself, which can be modeled with only minor changes to existing algorithms.

\section{Methods}

RBPBind incorporates protein-binding into secondary structure prediction using the paradigm of (Forties and Bundschuh 2010). Specifically, it computes the probability that an RBP will bind to an RNA through the use of partition functions, i.e.

$$
\mathbf{P}(\mathrm{RBP} \text { binds to RNA })=\frac{\sum_{\substack{\text { structures } U \\ \text { including an RBP }}} e^{-\beta \mathrm{E}(U)}}{\sum_{\text {all structures } S} e^{-\beta \mathrm{E}(S)}}
$$

where $E(U)$ is the energy of a particular protein-RNA structure, and by convention $\beta=\frac{1}{k_{B} T}$ where $k_{B}$ is Boltzmann's constant. In spite of the fact that the number of possible structures on both the top and bottom of Eq. (1) grows exponentially with the number of bases, the Vienna RNA package can compute such sums in the absence of proteins in polynomial time, using the recursive approach set forth in (McCaskill 1990). 
To incorproate RBPs, we express our partition function (the denominator of Eq. (1)) as

$$
Z(c)=Z_{0}+\sum_{i} Z_{i} \frac{c}{K_{d, i}^{(0)}}+\sum_{i<j} Z_{i j} \frac{c}{K_{d, i}^{(0)}} \frac{c}{K_{d, j}^{(0)}}+\ldots
$$

We assume that the protein binds to a fixed number of bases, say $m$, which then become unavailable for base-painring; this motivates our use of the quantity $Z_{i}$, which is the sum over all RNA structures in which nucleotides $i, \ldots, i+m-1$ are unpaired. We use $Z_{0}$ to represent the partition-function sum over all structures which have no protein attached.

The constant $K_{d, i}$ is called the bare dissociation constant at nucleotide $i$, and quantifies the RBP's tendency to bind to the nucleotides $i$ through $i+$ $m-1$ (provided these bases are unpaired). We also note that the numerator of 11 is given by $Z(c)-Z_{0}$, allowing us to evaluate the whole expression provided we have $Z(c)$. It was shown in (Forties and Bundschuh 2010. that this method of incorporating protein-binding into the partition function does not alter the $O\left(N^{3}\right)$ complexity of the calculation.

To arrive at $Z(c)$, however, we must obtain the bare dissociation constants $K_{d, i}^{(0)}$. Our methology in this task relies on relative dissociation constants derived from RNAcomplete experiments. Specifically, we obtain from the RNAcompete experiments a set of measurements $K_{d, i}^{(\mathrm{rel})}$ such that

$$
\gamma K_{d, i}^{(\mathrm{rel})}=K_{d, i}^{(0)}
$$

for a value $\gamma$ that is the same for every $i$. This allows us to evaluate the quantity $Z(\gamma c)$ for any $c$, using 2 with $K_{d, i}^{(\text {rel })}$ in place of $K_{d, i}^{(0)}$. To obtain the scaling factor $\gamma$, we then solve the equation

$$
\frac{Z(\gamma c)-Z_{0}}{Z(\gamma c)}=\frac{1}{2}
$$

for specific RNA-protein reactions whose effective dissociation constant have been established through in vitro experiments in the literature. Our solution $\gamma c$ then in principle equals the published effective dissociationconstants $K_{d \text {,eff }}$, and we perform a least squares procedure over several such reactions to obtain a final $\gamma$ (see Supplement for more details.)

\section{Interface}

The interface of RBPBind is straightforward. The user enters an RNA sequence of up to 250 bases, and chooses one of four available proteins. (These four proteins - HuR, RBFOX1, U2AF2 and KHDRBS3 - were selected based on the criterion that the two required data-types, relative binding constants from RNAcompete data and independent biochemical evidence for converting these into absolute binding constants as above were both available.)

The user submits the RNA and protein, and the server opens a window with the information shown in Fig. 11 a). This output window depicts the binding curve (the probability of an RBP being bound to the RNA as a function of protein concentration), as well as expected number of proteins bound to the RNA. The effective binding constant for the protein-RNA interaction is calculated as the concentration at which half of the RNA molecules are bound by protein.

RBPBind also computes information about site-specific binding Clicking a point on the graph, or entering a concentration in the box below it, will bring up a site-by-site profile, which plots the probability that protein will bind to any specific base. The plot in Fig. 1 b) gives the probability that the site will be covered by protein, i.e., that this site will lie anywhere in the footprint of a binding protein; the plot in Fig.11 (c) gives the probability that it will be occupied by protein, i.e., that this site is the one in the protein's footprint that is closest to the 5' end of the RNA. (a)

RNA protein interactions for TNFalpha45-example The apparent $K_{4}$ of HuR to your $\mathrm{K}$

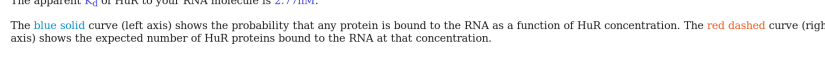

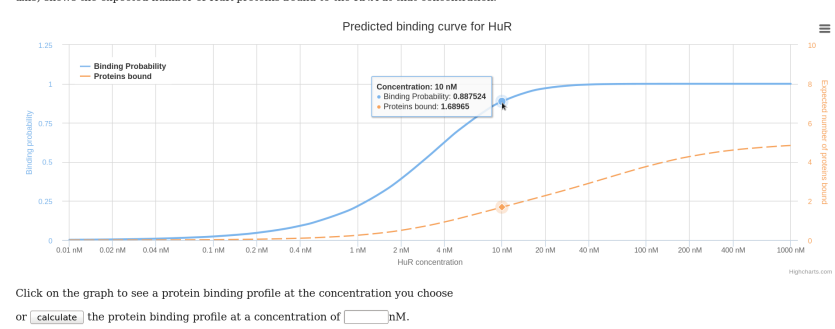

(b) Base coverage at $[\mathrm{HuR}]=10 \mathrm{nM}$

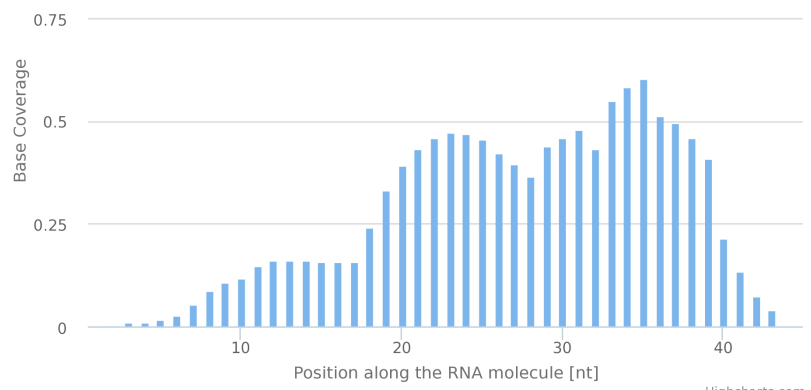

(c) Site occupancy at $[\mathrm{HuR}]=10 \mathrm{nM}$

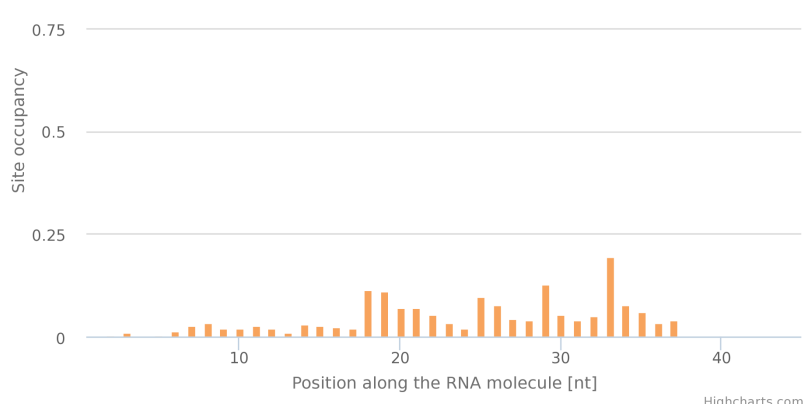

Fig. 1. Output of the RBPBind web server. When provided with an RNA sequence and choice of an RNA-binding protein, the web server initially responds with the effective $K_{d}$ for the RNA-potein interaction, the binding curve, and the expected number of proteins bound to the RNA as a function of protein concentration (a). After clicking on a point in the graph or manually entering a protein concentration, the sever further reports the probability that each base along the RNA is covered by a protein (b) and is the first base in the footprin of a protein (c).

\section{Conclusion}

We have introduced a server which provides researchers with a predicted affinity of any RNA for a set of single-stranded RNA binding proteins taking full account of all sites on the RNA as well as the protein's competition with the RNA's secondary structure. The server provides both the strength of the interaction and the expected binding locations. Given the importance of protein-RNA interactions we expect it to be of broad use for the RNA biology community. We anticipate adding support for additional proteins as more biochemical data becomes available. We also plan to extend our server to allow prediction of the simultaneous interactions of multiple proteins with an RNA, as well as to cover the case of double-stranded RBPs. 


\section{Acknowledgements}

We are grateful for useful discussions with Quaid Morris from the University of Toronto and for his kindness in sharing raw RNAcompete data and analysis code with us.

\section{Funding}

This material is based upon work supported by the National Science Foundation under Grants No. DMS-0931642 (JG) and DMR-1410172 (RB).

\section{References}

Forties, R. A. and Bundschuh, R. (2010). Modeling the interplay of single-stranded binding proteins and nucleic acid secondary structure. Bioinformatics, 26(1), 61-67.
Glisovic, T., Bachorik, J. L., Yong, J., and Dreyfuss, G. (2008). RNAbinding proteins and post-transcriptional gene regulation. FEBS letters, 582(14), 1977-1986.

Lambert, N., Robertson, A., Jangi, M., McGeary, S., Sharp, P. A., and Burge, C. B. (2014). RNA Bind-n-Seq: quantitative assessment of the sequence and structural binding specificity of RNA binding proteins. Mol Cell, 54(5), 887-900.

Lorenz, R., Bernhart, S. H., Honer Zu Siederdissen, C., Tafer, H., Flamm, C., Stadler, P. F., and Hofacker, I. L. (2011). ViennaRNA Package 2.0. Algorithms Mol Biol, 6, 26.

McCaskill, J. S. (1990). The equilibrium partition function and base pair binding probabilities for RNA secondary structure. Biopolymers, 29(67), 1105-1119.

Ray, D., Kazan, H., Chan, E. T., Castillo, L. P., Chaudhry, S., Talukder, S., Blencowe, B. J., Morris, Q., and Hughes, T. R. (2009). Rapid and systematic analysis of the RNA recognition specificities of RNA-binding proteins. Nature Biotechnology, 27(7), 667-670. 